\title{
Construction of luminosity function for galaxy clusters
}

\author{
Włodzimierz Godłowski ${ }^{1 *}$, Joanna Popiela ${ }^{1}$, Katarzyna Bajan ${ }^{2}$, \\ Monika Biernacka ${ }^{3}$, Piotr Flin ${ }^{3}$ and Elena Panko ${ }^{4}$ \\ ${ }^{1}$ Institute of Physics, Opole University, Opole, Poland \\ ${ }^{2}$ Institute of Physics, Pedagogical University, Cracow, Poland \\ ${ }^{3}$ Institute of Physics, Jan Kochanowski University, Kielce, Poland \\ ${ }^{4}$ Kalinenkov Observatory, Nikolaev State University, Nikolaev, Ukraine
}

\begin{abstract}
The luminosity function is an important quantity for analysis of large scale structure statistics, interpretation of galaxy counts ( Lin \& Kirshner 1996). We investigate the luminosity function of galaxy clusters. This is performed by counting the brightness of galaxies belonging to clusters in PF Catalogue. The obtained luminosity function is significantly different than that obtained both for optical and radiogalaxies (Machalski \& Godowski 2000). The implications of this result for theories of galaxy formation are discussed as well.
\end{abstract}

Keywords. galaxies:

\section{Introduction}

We construct and study the luminosity function ( Lin \& Kirshner 1996) of 6168 galaxy clusters. This was performed by counting the brightness of galaxies belonging to clusters in the magnitude range $m 3+3$ in PF Catalogue (Panko \& Flin 2006) taking data for galaxies from MRSS (Ungruhe, Seitter \& Durbeck 2003). The obtained luminosity function is significantly different than that obtained both for optical and radiogalaxies (Machalski \& Godłowski 2000). Our preliminary result obtained with Condon (1989) method shows that shape of Luminosity Function for galaxy clusters looks like Gauss function rather than a Schechter function.

\section{Conclusions}

Probable explanation of the diferences in the shape of the luminosity function of galaxy clusters and luminosity function of galaxy and radiogalaxy come from differences in scenarios of formation of galaxies and clusters.

\section{References}

Condon, J. J. 1989, ApJ 338, 13

Lin, H. \& Kirshner, R. P. 1996, ApJE 464, 60

Machalski, J. \& Godlowski, W. 2000, A\&A 360, 463

Panko, E. \& Flin, P. 2006 Journal Astron. Data, 12, 1

Ungruhe, R., Seitter, W. C., \& Durbeck, H. W. 2003, Journal Astronomical Data 9, 1 